smoking habit. A number of point-of-care urine cotinine tests are used to validate self-reported smoking and in some instances, provide feedback to improve smoking cessation. Urine testing is inappropriate in some instances, and while saliva testing is more acceptable, it is more difficult because cotinine is in lower concentrations compared to urine. A prototype saliva test was developed and evaluated, but the colorimetric assay was deemed inadequate. A new, more sensitive assay has been developed and evaluated in a group of healthy volunteers.

Method Volunteers $(n=117)$, aged between 22 and 67 years $(36 \%$ female), including 61 smokers with a cigarette consumption of five or more cigarettes/day, (mean 16.0), provided a saliva sample using a manufactured collecting device. One $\mathrm{ml}$ of saliva was eluted using the test's fixed-volume syringe. The sample was introduced onto freeze-dried reagents and quickly shaken. A sample positive for nicotine metabolites would be expected to turn pink within $1 \mathrm{~min}$, but 4 min were allowed for full colour development. The resultant colour was compared with a four-point colour chart and the level of smoking recorded. Samples from non-smokers should remain unchanged.

Results A positive colour change wasobtained from 56 of the 61 smokers and a negative result from 54 of the 56 non-smokers, giving a sensitivity of $92 \%$ and specificity of $96 \%$. The semi-quantitative results correlated with daily cigarette consumption; with light smokers (5-10 per day, $\mathrm{n}=15)$ mean $2.3,11-15$ per day $(\mathrm{n}=14)$ mean $2.8,16-20$ per day $(n=19) 3.4$ and more than 20 per day $(\mathrm{n}=8)$ mean $3.0(\mathrm{p}<0.05)$.

Discussion The new test was found to be superior to the prototype, being quicker and the final colour easier to read. The saliva collection device was also an improvement on previous methods. The sensitivity and specificity were comparable with the other commercial saliva cotinine test available. A dedicated colorimeter to quantify the result is under development. This test could be an important adjunct for treating smoking-related disease.

\section{P189 SMOKING STATUS PREDICTS BENEFIT FROM BREATHING RETRAINING FOR HYPERVENTILATION}

\author{
doi:10.1136/thx.2010.151043.40
}

C A Maguire, A G Robson, J Pentland, D McAllister, J A Innes. Respiratory Function Service and Dept of Physiotherapy, Royal Infirmary and Western General Hospital, Edinburgh, UK

Introduction Hyperventilation syndrome has a prevalence of $6-11 \%$ in primary care, and can be treated via breathing retraining. Breathing retraining reduces hyperventilation and improves symptoms. However, it is staffing intensive. Therefore, we examined which patient characteristics are associated with benefit from breathing retraining.

Method Retrospectively, we identified 201 consecutive patients referred to the breathing retraining service (February 2003 to June 2009) at a single site. Treatment efficacy was assessed by the treating physiotherapist according to resolution of symptoms. Success was defined as complete or near complete resolution of symptoms at the end of the breathing retraining period. Height, age, sex, smoking status, ethnicity, hyperventilation type (acute or chronic), restrictive/obstructive spirometry and known cardio/ respiratory disease were also recorded. Patient characteristics were compared by treatment efficacy using $\chi^{2}$ tests and $t$-tests, and logistic regression was used to identify which characteristics were independently associated with treatment efficacy.

Results The mean (SD) age was 50.9 (15.5) years. 38.3\% were male and $31 \%$ had acute hyperventilation. $15.9 \%$ had obstructive and $10.5 \%$ had restrictive spirometry. $46.3 \%$ had known cardiovascular or respiratory disease. 61 patients overall benefited from breathing retraining. Current smokers were much less likely to benefit from breathing retraining compared to non-smokers ( 1 in 16.5 vs 1 in 2.4, $\mathrm{p}=<0.01)$. This association persisted after adjusting for the above patient characteristics. Ex-smokers had a similar probability of benefiting to that of non-smokers (1 in 3). Known cardiorespiratory disease was also independently associated with a lower odds of benefiting. The ORs for successful breathing retraining are shown for each predictor in the Abstract P189 Table 1. None of the remaining characteristics were associated with treatment efficacy.

\section{Abstract P189 Table 1}

\begin{tabular}{llll}
\hline Characteristic & $\begin{array}{l}\text { OROR (Odds of benefit } \\
\text { from breathing retraining) }\end{array}$ & $\mathbf{9 5 \%}$ Cl of 0R & p Value \\
\hline Age (per 10 years) & 1.12 & 0.87 to 1.45 & 0.39 \\
Men & 0.37 & 0.13 to 0.99 & 0.05 \\
Nijmegen score & 0.97 & 0.94 to 1.01 & 0.11 \\
Cardio/respiratory disease & 0.49 & 0.24 to 0.98 & 0.05 \\
Spirometry & & & 0.22 \\
$\quad$ Obstructive & 2.41 & 0.88 to 6.63 & \\
$\quad$ Restrictive & 1.07 & 0.33 to 3.16 & \\
Smoking status & & & $<0.001$ \\
$\quad$ Ex-smoker & 0.60 & 0.27 to 1.33 & \\
$\quad$ Current smoker & 0.08 & 0.01 to 0.33 & \\
\hline
\end{tabular}

Conclusion Our sample size was comparatively small as reflected in the wide CI, and the outcome measure was subjective. Nevertheless, smoking status is strongly associated with physiotherapistassessed treatment efficacy following breathing retraining. Smokers, but not-ex-smokers are much less likely to benefit from breathing retraining. Therefore referral for smoking cessation rather than breathing retraining may be more appropriate in this patient group. It was not possible to assess long term benefit from this retrospective study.

\section{P190 ATTITUDES OF HEALTH CARE PROFESSIONALS TOWARDS SMOKING CESSATION}

doi:10.1136/thx.2010.151043.41

S Pearce, J Maycock, C McCauley, D Nazareth, P Stockton. St Helen's and Knowsley Teaching Hospitals NHS Trust, Prescot, UK

Background NICE (UK) recommends that all healthcare professionals (HCPs) refer patients who would like to stop smoking to an NHS Stop Smoking Service (SSS). ${ }^{1}$ This study explores attitudes of HCPs and factors that may contribute to a low referral rate to SSS Methods 164 HCPs (83 doctors, 72 nurses, 9 pharmacists) completed a structured questionnaire exploring reasons as to why they would not refer to smoking cessation services.

Results Smoking cessation was considered to be an important health issue for $95 \%$ of respondents, however only $51 \%$ routinely asked smokers if they wanted to quit. 37\% were not familiar with smoking cessation guidelines (local or national). $40 \%$ supported a formal referral system involving a GP and $55 \%$ would like more training. The main reasons for NOT referring to SSS are outlined below.

Conclusions The vast majority of HCPs considered smoking cessation to be an important issue. However, a significant proportion of HCPs were unaware of local/national guidelines. This appears tobe a significant barrier to the referral of patients to SSS. Most HCPs would like further targeted training and information. Since this survey the Trust has modified the generic Trust Admission Proforma to prompt HCPs to consider referring to SSS. Teaching sessions have been introduced for HCPs to enable them to deliver accurate stop smoking information to smokers. 